\title{
Long Stokes shifts and vibronic couplings in perfluorinated polyanilines
}

\author{
Panagiotis Dallas, ${ }^{a^{*}}$ Ilija Rašović, ${ }^{a}$ Tim Puchtler, ${ }^{b}$ Robert A.Taylor ${ }^{b}$, and Kyriakos Porfyrakis ${ }^{a}$ \\ a. Department of Materials, University of Oxford, $\mathrm{OX1} 3 \mathrm{PH}$, United Kingdom. \\ b. Clarendon Laboratory, Physics Department, University of Oxford, United Kingdom. \\ † panagiotis.dallas@materials.ox.ac.uk; Tel: +44(0)1865283342.
}

We report the effect of surfactant addition on the optical properties of perfluorinated polyanilines synthesized through liquid-liquid interfaces. We obtained very long Stokes shifts, $205 \mathrm{~nm}$, for oligomers derived from a hydrofluoroether-water system in the presence of Triton X-100 as a surfactant, and vibronic fine features from a toluene-water system.

Light emitting polymers have found numerous applications in biomedicine ${ }^{[1]}$, as components of light emitting diodes ${ }^{[2]}$ and as optical sensors. [3] An interesting class of fluorescent polymers are the fluorinated polyphenylenes that exhibit unusually high quantum yields in the region of $68 \%$. ${ }^{[4]}$ This very bright blue fluorescence was attributed by the authors to the presence of electron withdrawing fluorine groups that also have the ability to reduce the HOMO and LUMO levels making the polymers suitable for electro-optic devices. Among the various methods for the synthesis of conjugated and/or conductive polymers, interfacial complexation/polymerization emerged as an important strategy for the synthesis of photo functional single layers [5], nanofibers [6-7], fluorescent perfluorinated polymers [8], and noble metal nanocomposites [9], with polyaniline nanostructures proven to be extremely efficient gas sensors ${ }^{[10]}$. In a previous work by Dallas et al. ${ }^{[8]}$ the light emitting properties of perfluorinated oligoanilines based on 3perfluoroctyl aniline were rationalized through structural differences in the polymer backbone, variations of which were synthesized through five different organic-aqueous interfaces with interfacial tensions ranging from 28 to $52 \mathrm{mN} / \mathrm{m}$. ${ }^{[11]} \mathrm{A}$ series of materials have been synthesized through interfaces, examples include nanoscale mirrors ${ }^{[12]}$ and DNA driven nanoparticle arrays ${ }^{[13]}$, while the absorption and desorption of ions in interfaces has been utilized towards the control of nanomaterials assembly. ${ }^{[14]}$ In order to reduce and control the surface forces, a series of surfactants have been employed, even for unconventional systems and niche applications such as supercritical $\mathrm{CO}_{2}$-water [15] and fluorocarbon-water, alongside the conventional water-hydrocarbon system. ${ }^{[16]}$

In this work we focused our study on two different liquid-liquid interfacial polymerization systems, toluene-water and hydrofluoroether (NOVECTM 3M 7300; HFE)-water for the polymerization of 4-perfluoroctyl aniline, with the addition of two different surfactants, Triton X-100 and triethylene glycol diethyl ether. We performed FTIR/Raman and ${ }^{1} \mathrm{H}$ and COSY NMR spectroscopic analyses in order to identify the molecular structure of the products. Interfacial polymerizations in dichloromethane or chloroform as the organic phases were also conducted. Unique vibronic features were observed in the fluorescent oligomers derived from a toluene-water interface and we elucidated the structure of two core oligomers. This interesting observation was coupled with an unusually high red shifted photoluminescence pattern observed for the samples synthesized from HFE-water system with the addition of surfactant. For the latter case, the fraction with the highest Stokes shift showed a $\lambda_{\mathrm{em}}=575 \mathrm{~nm}$ at a $\lambda_{\mathrm{ex}}=370 \mathrm{~nm}$, one of the longest Stokes shifts reported to date.

It is expected that organic solvents with significantly variable interfacial tension and characteristics will lead to differences in the oxidation and protonation states and molecular weight of the synthesized polymers. In order to tune the characteristics of the interface, the surfactant employed was Triton X-100 with a critical micelle concentration of $0.25 \mathrm{mM}(0.162 \mathrm{mg} / \mathrm{ml})$. To that end, the polymerization took place at concentrations below (toluene-water with $0.125 \mathrm{mg} / \mathrm{ml}$ of surfactant) and above the critical micelle concentration ( 1.25 and $12.5 \mathrm{mg} / \mathrm{ml}$ for toluenewater and $1.75 \mathrm{mg} / \mathrm{ml}$ for HFE-water system). When the more hydrophilic triethylene glycol was used instead of Triton, the polymerization proceeded at a significantly slower rate and did not lead to a stable film on the interface; instead we obtained an amorphous precipitate, probably due to its reducing agent character and hydrophilicity. We confirmed the effect of 
surfactant in the crystal packing of the thin films through Raman spectroscopy (Fig.S9). The monomer demonstrated a spectrum implying higher crystallinity. However, in the materials synthesized with Triton, three new peaks with relatively strong intensity appear in the $\mathrm{C}-\mathrm{N}$ region, at 1148; 1402 and $1462 \mathrm{~cm}$ 1. Furthermore, the films synthesized without surfactant and below CMC had identical Raman peaks, similar for the two samples above the CMC. The polymerization reaction responsible for the formation of polyaniline chains is an electrophilic substitution. The attacking molecule is oxidized and acts as an electrophile. ${ }^{[17]}$ We have to consider that strongly electron withdrawing groups like $\mathrm{C}_{7} \mathrm{~F}_{15}$ favour electrophilic attack to the meta position. On the other hand, a strongly activating group such as $\mathrm{NH}_{2}$ directs the electrophile to the ortho or para position. In the perfluorinated aniline the para position is occupied by the perfluorinated chain. The ortho position for the $\mathrm{NH}_{2}$ is identical with the meta position for the electron deactivating group so we suspect that the substitution occurs favourably at the proton next to the $\mathrm{NH}_{2}$ as indicated in Fig.1b. Fig.1 shows the different orientations of a surfactant in the hydrocarbon-water and the fluorocarbon-water interface. In general fluorocarbons have a much weaker affinity with water compared to hydrocarbons, hence we assume that the amphiphilic arrangement of Triton on the HFE/water interface will be in a less dense arrangement compared to the toluene or chloroform-water. [18] MALDI-TOF experiments (Fig.S7-8) demonstrated a different fragmentation and ionization for the HFE derived samples with fragments corresponding to 5 or 6 sub units while for the toluene derivatives, no fragments with more than 3 or 4 subunits appeared.

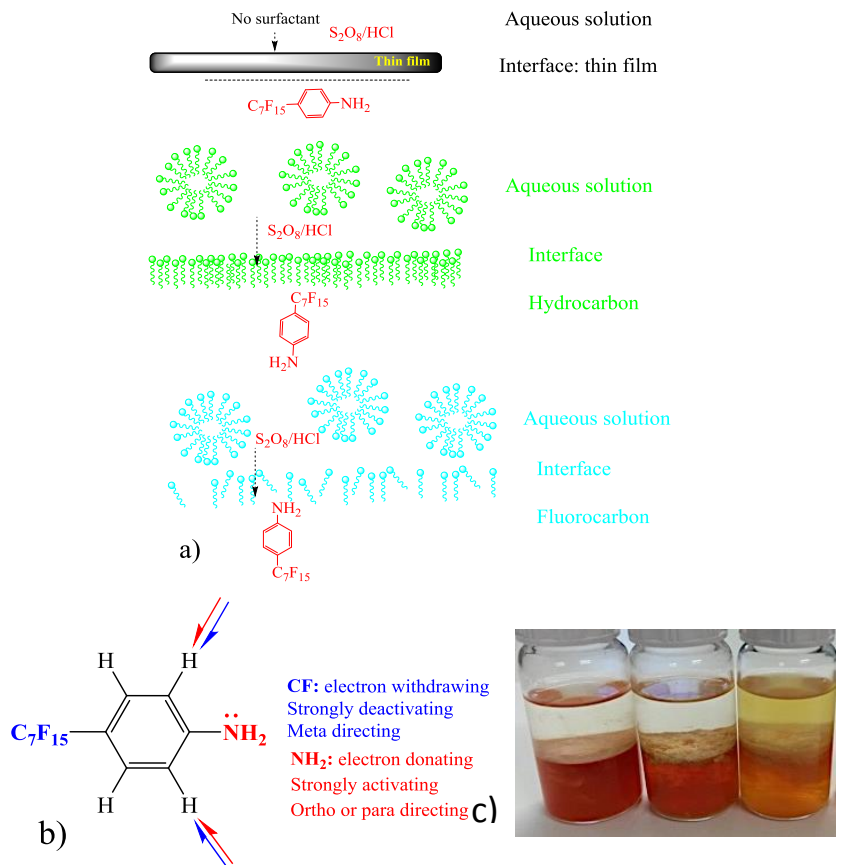

Fig.1. a) A schematic representation for the formation of micelles and the interface structure when no surfactant is added; when it is added in a hydrocarbon-water system; and in a fluorocarbon-water system. b) Promoted positions of electrophilic attack on 4-perfluoroctyl aniline. c) The interfacial polymerization from an HFE-water system with, from left to right, the following amounts of monomer $(x)$, acid (y), oxidant (z), surfactant (s): a) $x=240 \mathrm{mg}, y=0.1 \mathrm{ml}, \mathrm{z}=240 \mathrm{mg}, \mathrm{s}=14$ $\mathrm{mg}$; b) $\mathrm{x}=240 \mathrm{mg}, \mathrm{y}=0.3 \mathrm{ml}, \mathrm{z}=720 \mathrm{mg}, \mathrm{s}=50 \mathrm{mg}$; ) $\mathrm{x}=240$ $\mathrm{mg}, \mathrm{y}=0.3 \mathrm{ml}, \mathrm{z}=720 \mathrm{mg}, \mathrm{s}=68 \mathrm{mg}$. Notice the migration of greenish oligomers to the aqueous phase in (c).

After the completion of the reaction and filtration of the films, we then proceeded to separate the soluble oligomers through silica column chromatography. We evaporated the reaction solvent after filtration and again dissolved the oligomeric materials in toluene since it has the optimum interaction with silica. We initially characterized the soluble fractions with UVvis spectroscopy and the spectra are shown in Figure $2 \mathrm{~b}$ for all the toluene-water derivatives. We present the different isolated, soluble fractions when $0,1,10$ and $100 \mathrm{mg}$ of Triton X100 was added in the aqueous phase. When small chlorocarbon solvents such as dichloromethane and chloroform were tested, they resulted in polymers with very small Stokes shifts. FTIR spectroscopy provided a first indicative picture on the structure of the materials. The $\mathrm{NH}_{2}$ bending vibration appears with strong intensity at $1630 \mathrm{~cm}^{-1}$ for the monomer but with substantially lower intensity for the oligomers, due to the significantly lower percentage of unreacted $\mathrm{NH}_{2}$ groups in the fractions. Furthermore, the peak splits to weak vibrations at 1637, 1620, $1610 \mathrm{~cm}^{-1}$, signalling different $\mathrm{N}-\mathrm{H}$ environments, in accordance with NMR observations (Fig. 2-3 and S10). When Triton X-100 was added in the toluene-water system, due to its affinity with the toluene phase it migrated to the organic phase and acted as a counterbalancing anion. We were able to confirm this from the strong symmetric and asymmetric $-\mathrm{C}-\mathrm{H}$ vibrations in the FTIR spectrum accompanied by the absence of the $-\mathrm{OH}$ vibration, signalling the presence of anionic oxygen group as counter anion.

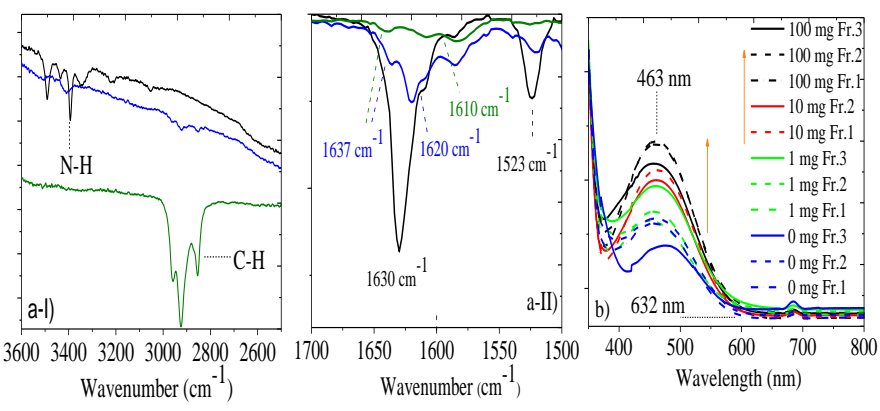

Fig.2. a-I/II) FTIR spectra for the monomer (black); the soluble oligomeric fraction derived from HFE-water with Triton (blue); and the soluble oligomeric fraction derived from toluene-water with $100 \mathrm{mg}$ Triton (green). b) UV-visible spectra for all the water-toluene fractions.

The ${ }^{1} \mathrm{H}$ NMR spectra for two representative samples derived from toluene-water systems are presented in Fig.3. As discussed, the preferred available site of electrophilic attack is the ortho- position relative to the amine group. We suggest a step-growth reaction mechanism which, for the sample from water-toluene Fr.1-no Triton, culminates in an oligomer 
consisting of five monomer units and incapable of polymerising further due to steric hindrance. The material is in the fully oxidised pernigraniline form. In addition to more peaks in the aromatic region (7.93;7.91-7.59;7.54;7.53-7.33;7.31;7.29; ppm), the sample from a Triton addition displays an extra peak at $9.72 \mathrm{ppm}$ which has been repeatedly assigned to the amine group of a leucoemeraldine structure in conventional aniline oligomers. [19] Unfortunately, the samples derived from HFE present much more complicated spectra (see S.I). The abundance of unique aromatic proton environments indicates that a variety of cross linked structures are present. Furthermore, while only one peak for terminal $-\mathrm{NH}_{2}$ groups can be seen in the toluene derived samples, the HFE derivative exhibits three terminal amine peaks, a further indication that an $-\mathrm{NH}_{2}$ group will further red shift the emission. These findings are in good agreement with the MALDI-TOF data indicating increased cross-linking for the HFE derivatives.
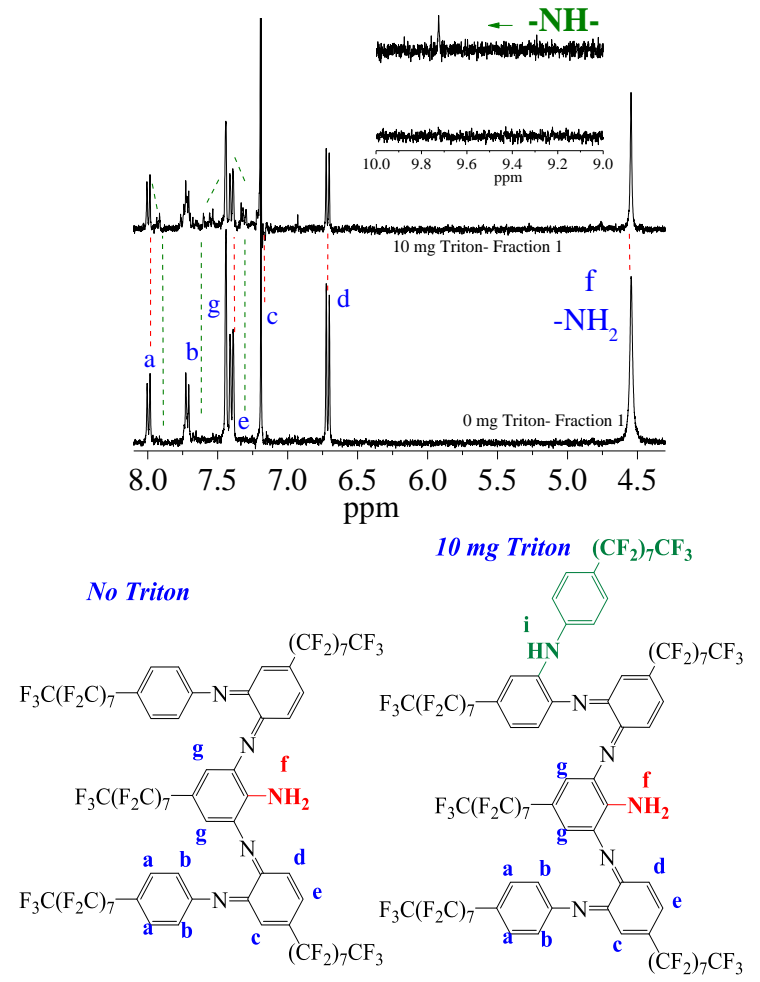

Fig.3. ${ }^{1} \mathrm{H}$ NMR data for samples derived from toluene without surfactant, and from toluene with the addition of $10 \mathrm{mg}$ Triton (Fr.1). We assign two core oligomers, a fully oxidized pernigraniline and a further cross-linked structure. The 2-D COSY NMR (Fig.S10) supports the structure assigned in the ${ }^{1} \mathrm{H}$ NMR. The doublets of protons $a$ and $b$ are coupled in the same benzene ring, as are the doublets of protons $d$ and $e$.

The excitation dependent photoluminescence (PL) maps from dichloromethane, chloroform, toluene and hydrofluoroetherwater systems in the absence of any surfactants are shown in supporting information section. In Fig. 4 we present the PL maps for the toluene-water system with the addition of Triton. We chose to focus on the toluene and HFE systems since they exhibit the longest Stokes shift and unique vibronic features in the PL spectra, of greatest interest for our work. While the three isolated fractions from the system without any surfactant (S.I) demonstrate only a weak ultraviolet emission, we observe an interesting trend in the toluene-water/Triton systems with the addition of Triton. The first fraction with low retention time exhibits a red shifted pattern with pronounced vibronic features, which nearly disappears in the second fraction of higher retention time. The quantum yield ( $Q Y$ ) for the fraction exhibiting the unique vibronic features from toluene solution was calculated to be $2.2 \%$; for the sample with vibronic features derived from HFE (Fr.1) it was $5 \%$; while for the sample with the exceptionally long Stokes shift, derived also from HFE, the QY was significantly lower, $0.5 \%$ (see Figure S11). Finally, the molar extinction coefficient was calculated using pyrene as a reference for the Fraction 1 from toluene (Figure S13). For the maximum absorbance located at the ultraviolet region it was 4536 and for the visible region absorbance ( $n \rightarrow p^{*}$ transition), $687 \mathrm{~cm}^{-1} / \mathrm{M}$. The concentration was $0.26 \mathrm{mg} / \mathrm{ml}(0.085 \mathrm{mM})$.
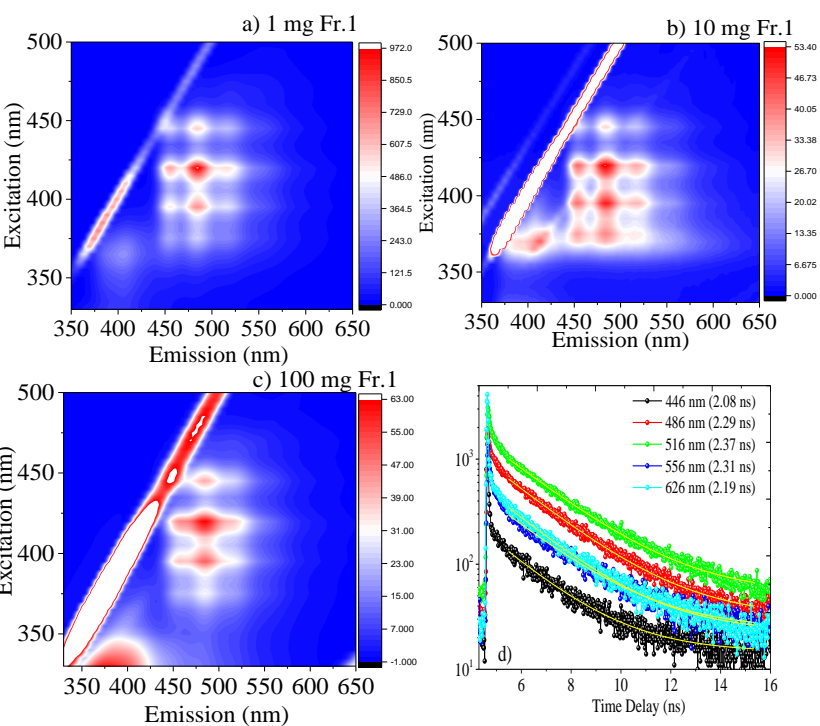

Fig.4. Excitation dependent photoluminescence maps for the toluene derived first fractions, when synthesized with the following amounts of Triton X-100 (a) $1 \mathrm{mg}$ (b) $10 \mathrm{mg}$ (c) $100 \mathrm{mg}$. d) Time resolved fluorescence for the sample (b), Fraction 1 . The lifetimes are indicated in parentheses.

While the first fraction derived from the hydrofluoroether (Fig.5), in the presence of Triton demonstrated a pattern with strong vibronic couplings, similarly with toluene derived sample separated by 1281 and $1414 \mathrm{~cm}^{-1}$ (Table 1 in S.I.;), for the second fraction, unusually high Stokes shift of more than 200 $\mathrm{nm}$ where observed. Typical values for other organic fluorophores are in the range of $90 \mathrm{~nm}$, for example carbon dots with blue emission, ${ }^{[20]}$ with many red fluorescent dyes currently finding applications in cell labelling, ${ }^{[21]}$ while the tailored fluorophores synthesized from 9-(acylimino)- and 9(sulfonylimino)pyronin have exhibited Stokes shifts in the range of $200 \mathrm{~nm}$. ${ }^{[22]}$ On the other hand inorganic quantum dots like $\mathrm{CuInS}_{2} / \mathrm{ZnS}$ QDs possess the dual advantages of unusually high quantum yields in the region of $81 \%$ and large Stokes shifts of more than $150 \mathrm{~nm}$ and osmium complexes show Stokes shifts of $320 \mathrm{~nm}$. [23a-b] While the first fraction consists clearly from a 
single fluorophore, from the PL maps it appears that the Fr.2 and Fr.3, probably consist from a strongly fluorescent and additional weekly fluorescent components. The decay lifetimes recorded for three representative samples: the first fraction of the toluene-Triton system with strong vibronic features, and the first two fractions derived from HFE. The toluene-Fr.1 and the HFE.Fr2 samples demonstrate an ultrafast first component $\left(\mathrm{S}_{2}\right.$ to $S_{1}$ transition) and a subsequent monoexponential decay from $\mathrm{S}_{1}$ to $\mathrm{S}_{0}$. The longest lifetime was derived from the first HFE fraction and it was $2.65 \mathrm{~nm}$ at a $\lambda_{\mathrm{em}}=596 \mathrm{~nm}$. Similar ultrafast biexponential dynamics and vibronic features observed in micellar and vesicle solutions of fluorophores, such as coumarin 153. [25-26] A monoexponential decay was observed for the HFE.Fr.1 sample with similar lifetimes.

a)
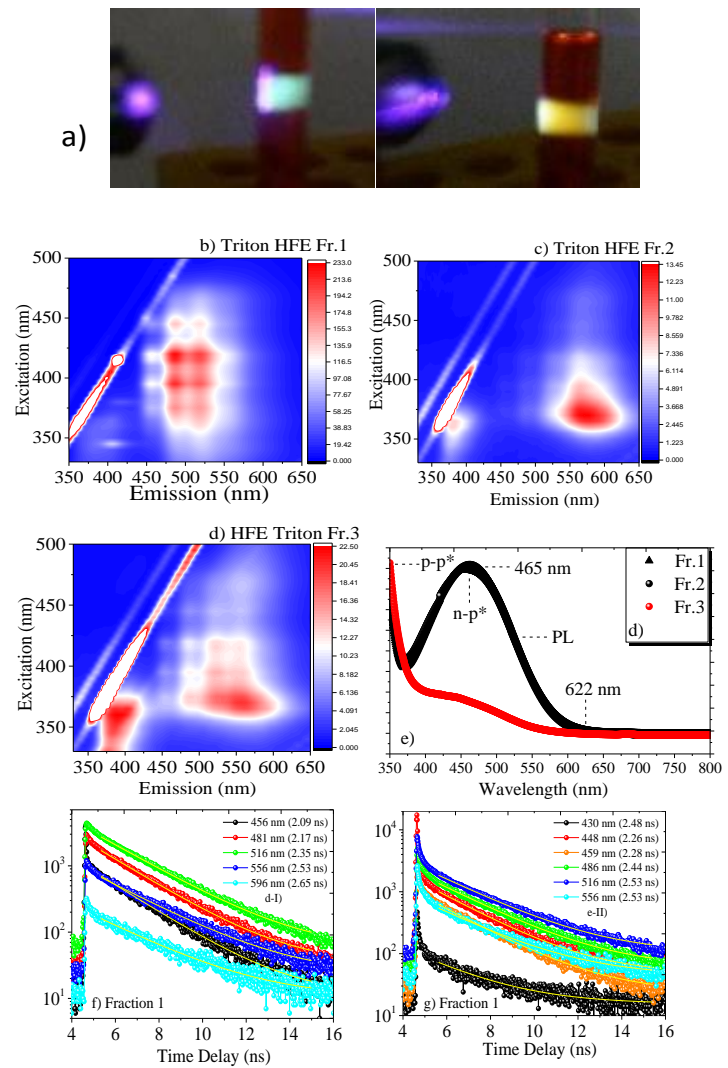

Fig.5. a) Images under $400 \mathrm{~nm}$ laser irradiation for Fr.1 (greenish) and Fr.2 (yellowish). (b-d) PL maps and the UV-Vis spectra (e) for the three separated fractions of the HFE-waterTriton system. The strong band at $465 \mathrm{~nm}$ is associated with conjugated aromatic amine polymers ( $n-p^{*}$ transition). ${ }^{[24]} f-g$ ) Time resolved fluorescence for the first two fractions.

In summary, we have seen large Stokes shifts and distinctive vibronic features and fine structures in the fluorescence of perfluorinated polyanilines through the incorporation of Triton $X-100$, in the aqueous phase against a perfluorinated ether and toluene respectively. We identified two distinctive core oligomeric units responsible for the fine features in the fluorescence spectrum while a further cross linked structure with amine pendant units is responsible for the red shifted emission.

\section{Notes and references}

¥ We acknowledge EPSRC (grant number: EP/K030108/1). We acknowledge Mr Georgios Velkos for his help during the synthesis and purification.

\section{References}

1 L.D.Lavis, R.T.Raines. ACS Chem.Biol. 2014, 9, 855

2 C.H.Chou, M.H.Hsu, F.C.Chen. Nano Energy 2015, 15, 729-736

3 K.P. Carter, A.M. Young, A.E. Palmer. Chem.Rev. 2014, 114 (8), 4564-4601

4 U.Giovanella, C.Botta, F.Galeotti, B.Vercelli, S.Battiato, M.Pasini. J.Mater.Chem.C. 2013, 1, 5322-5329

5 R.Sakamoto, K.Hoshiko, Q.Liu, T.Yagi, T.Nagayama, S.Kusaka, M.Tsuchiya, Y.Kitagawa, W.Y.Wong, H.Nishihara. Nat.Commun. 2015, 6, 6713

6 J.Huang, R.B.Kaner. Nat.Mater. 2004, 3, 783-786

7 J.X.Huang, S.Virji, B.H.Weiller, R.B.Kaner.J.Am.Chem.Soc. 2003, 125, 314-315.

8 P.Dallas,I.Rasovic,K.Porfyrakis. J.Phys.Chem.B. 2016, 120(13), 3441-3454

9 P.Dallas, D.Niarchos, D.Vrbanic, N.Boukos, S.Pejovnik, C.Trapalis, D.Petridis. Polymer. 2007, 48, 2007-2013

10 I.Fratoddi, I.Venditti, C.Cametti, M.V.Russo. Sensor.Actuat.B. 2015, 220, 534-548

11 P.Dallas, V.Georgakilas Adv.Coll.Int.Sci. 2015, 224, 46-61

12 V.A.Turek, M.P.Cecchini, J.Paget, A.R.Kucernak, A.A.Kornyshev, J.B.Edel. ACS Nano 2012, 6, 77897799

13 S.Srivastava, D.Nykypanchuk, M.Fukuto, J.D.Halverson, A.V.Tkachenko, K.G.Yager, O.Gang. J.Am.Chem.Soc. 2014, 136(23), 8323-8332

14 S.G.Booth, R.A.W.Dryfe. J.Phys.Chem.C. 2015, 119, 23295 23309

15 P.Chiquet, J-L.Daridon, D.Broseta, S.Thibeau. Energ.Convers. Manage. 2007, 48, 736-744

16 A.Bahramian, A.Danesh. Fluid Phase Equil. 2004, 221, 197-205

17 H.D.Tran, J.M.D'Arcy, Y.Wang, P.J.Beltramo, V.A.Strong, R.N.Kaner. J.Mater.Chem. 2011, 21, 3534-3550

18 P.Mukerjee, T.Handa. J.Phys.Chem. 1981, 85, 2298-2303

19 a) X.Ma, J.Ge, Y.Li, B.Guo, P.X.Ma. RSC Adv. 2014, 4, 1365213661 b) J.Kr ${ }^{2} \mathrm{iz}$, L.Starovoytova, M.Trchova, E.N. Konyushenko, J.Stejskal. J.Phys.Chem. B 2009, 113, 6666-6673

20 A.B.Bourlinos, A.Stassinopoulos, D.Anglos, R.Zboril, V.Georgakilas, E.P.Giannelis. Chem.Mater. 2008, 20, 45394541

21 AN. Butkevich, G.Y.Mitronova, S.C. Sidenstein, et al. Angew.Chem.Int.Ed. 2016, 55, 3290.

22 P. Horvath, P.Sebej, T.Šolomek, P.Klan. J. Org. Chem. 2015, 80 , 1299-1311

23 a) C.Li, W.Chen, D.Wu, D.Quan, Ziming .Zhou, J.Hao, J.Qin, Y.Li, Z.He, K.Wang. Sci.Rep. 2015, 5, 17777. b) C.Zhu, S.Li, M. Luo, et al. Nat. Chem. 2013, 5, 698.

24 K.Jiang, S.Ma, Y.Zhang, X.Han. RSC Adv. 2016, 6, 21895

25 N.Sarma, J.M.Borah, S.Mahiuddin. J.Phys.Chem. B. 2011, 115, 9040-9049

26 N.Sarkar, A.Datta, S.Das, Kankan Bhattacharyya. J. Phys. Chem. 1996, 100, 15483-15486 